\title{
Evaluation of correlation between anthropometric characteristics and scoliosis deformity of male students
}

\author{
Masoumeh MeHRABI*1 ${ }^{*}$, Khalill ABdOLlahi ${ }^{2}$, Dr.Ahmad HeMATFAR ${ }^{3}$, \\ Mohammad ABDOLLAHI ${ }^{4}$ \\ 1. Department of Physical Education \& Sport Sciences, Islamic Azad University of Broujerd \\ (Corresponding author). E-mail: masoumeh.mehrabi2012@yahoo.com . \\ 2. Department of Physical Education \& Sport Sciences, Islamic Azad University of Karaj \\ 3. Assistant Professor, Department of Physical Education \& Sport Sciences, Islamic Azad \\ University of Broujerd \\ 4. Department of Physical Education \& Sport Sciences, Islamic Azad University of Broujerd
}

\begin{abstract}
Introduction: The aim of this study was to evaluate of correlation between anthropometric characteristics and scoliosis deformity of male students.

Methodology: The scoliosis was studied in 28 male students with a vertical line with the subjects standing in a relaxed position. Then each subject was tested for various anthropometric measurements, a set of anthropometric measurements, which included height, body weight, body composition and muscle mass and etc. Statistical analysis was performed with use of SPSS statistical software. Pearson correlation coefficient, with significance levels of 0.05 , was performed to assess correlation between variables.

Results: Results didn't show any significant correlation $(\mathrm{P}>0.05)$ between scoliosis and anthropometric characteristics.

Conclusion: Scoliosis didn't show any significant correlation with anthropometric characteristics, however further studies are needed to prove this.
\end{abstract}

Keywords: Scoliosis, Anthropometric, Correlation

\section{Introduction}

Malformation of the spine are often visible as scoliosis, lordosis, and kyphosis. (1).The lateral deviation of the spine is called scoliosis that are two forms of $s$ and c. (1).Despite the development of medical science, Industry growth and appearance of machines, cars, and technology in people's lives, and the changes in the lifestyle of modern people, discomforts and new diseases such as postural malformation threaten some people's life (2). Postural abnormality changes are adverse changes that disrupt the structure of skeletal and postural alignment. If the abnormality is not detected and treated on time, it may have diverse effects on physiological function, e g scoliosis disorders:

Pain, walking difficulty, decrease of the spine mobility, neurological disorders, pressure on the nerve roots, reduction of vital capacities cardio respiratory system dysfunction, decrease of the spine mobility and unpleasant appearance (3). Postural abnormalities, except hereditary 
factor, occur due to life industrialization, inactivity, wrong habits, and the use of nonstandard equipment (4).

Man has different physical positions day and night which some of these cases cause lesions and abnormalities because of the long term repeatetive positions (5). As well as, noncomplince with ergonomic standards of continous use of equipment and the structural dismatch between the anthropometric characteristics of users can cause physiological disorders. The former researches reported the high prevalence of abnormalities in the community of university students. Mahdavinejad reported the high prevalence ( 90 percent) of the physical malformation incidence among male students and about the complications of the spine, the most malformations in order are down shoulder, lordosis, swelling of the abdomen, protruding shoulder (6). In a survey of upper limb malformations of Martyr Chamran university students , 58\% lumbar lordosis,32\% cervical lordosis ,24\% crooked neck, $14 \%$ crooked neck, $14 \%$ kyphosis, and 3\% scoliosis were reported among male ones (7).Rahmani and his colleagues in 2012 studied about the relationship between flexibility and body mass index with spine malformations in people with Down syndrome. The results showed a significant relationship between body mass index and lordosis (8).shape change occurs under the influence of various factors. There isn't a clear and definitive scientific link between physical malformations featuring anthropometric characteristics. On the other hand, now a huge part of our society consists of university students.

It seems that special attention to their physical and mental health through malformations reviews of the spine is very crucial and it needs to be more and more.

Therefore, this study examine the relationship between scoliosis malformations featuring anthropometric characteristics among physical education students.

\section{Methodology}

This study is the semi-empirical .The sample in this study included 28 men of P.E students with an average age of $25 / 96$, height of $177 / 29 \mathrm{~cm}$ and weight of $80 / 65 \mathrm{~kg}$ who were selected as subjects in this study. After a full explanation of the design of the research, the subjects, who volunteered to be analyzed for their spinal abnormalities, filled out demographic questionnaire and testimonial. To detect scoliosis, we passed a plumb line from the seventh cervical vertebrae along the spinal process of participants. The line must be along all the spinal processes. The line, which was said, does not pass through the spine of people with scoliosis. In this case, the distance of the line from the spine measured and recorded (2).

For greater certainlity, each measurement was reported twice and the average angle was obtained. To measure the height $(\mathrm{cm})$ of the device (seca) made in Germany was used. For This purpose, the participants were asked to attach to dial without shoes from behind while standing in a way that shoulders, hip, calf and heels are in contact with the dial and legs should be placed next to each other. In addition, the upper part of the external auditory canal must be parallel with cheekbone and individuals must look to the face while measuring their height. To determine the height while sitting, the subject on the couch is against the wall, sitting back against the wall and then the distance between the top of the head on the wall opposite to the couch was measured by tape and recorded. To measure body weight, body 
mass index(BMI), fat percentage, muscle mass percentage and visceral fat in the body, the device of the body composition BF511 made in Japan, was used.

For this purpose, the subjects were asked to take off their thick clothes, heavy ornaments, and their shoes as well. While their legs are placed in designated areas on the screen, they pull out the levers of the device in front of their chest completely in a way that 8 sensors of the device must be activated. They remain motionless for a few seconds then all the information appears on the monitor in digital form that can be recorded. Upper limb length measured by determining the distance between excrescence stretched and the tip of the middle finger while hand was fully stretched and opened. Kolmogorof-smirnof's test was used for checking normality distribution to describe and analyze statistically and Pearson's correlation coefficient was used for this purpose. All operations were performed by using SPSS16 software $\&$ the significance lever for all calculation was considered $(\mathrm{P}<0 / 05)$.

\section{Results}

The results showed that the highest value is the correlation between age and scoliosis deformity.

However, the results of correlation coefficient showed a significant association between variables (Table 1) Trend line graph between the scoliosis and muscle mass was given in figure 1.

Table1: Checking the correlation between the scoliosis with anthropometric characteristics.

\begin{tabular}{|l|c|c|c|}
\hline & NUMBER & CORRELATION(r) & SIGNIFICANCE LEVEL(P) \\
\hline Age and scoliosis & 28 & -0.322 & 0.126 \\
\hline Standing height with scoliosis & 28 & 0.214 & 0.316 \\
\hline Setting height with scoliosis & 28 & 0.237 & 0.265 \\
\hline Weight with scoliosis & 28 & -0.026 & 0.904 \\
\hline Arms legs with scoliosis & 28 & 0.128 & 0.550 \\
\hline Fat percentage with scoliosis & 28 & 0.227 & 0.286 \\
\hline Scoliosis with BMI & 28 & -0.182 & 0.394 \\
\hline Scoliosis with muscle mass & 28 & 0.239 & 0.261 \\
\hline
\end{tabular}




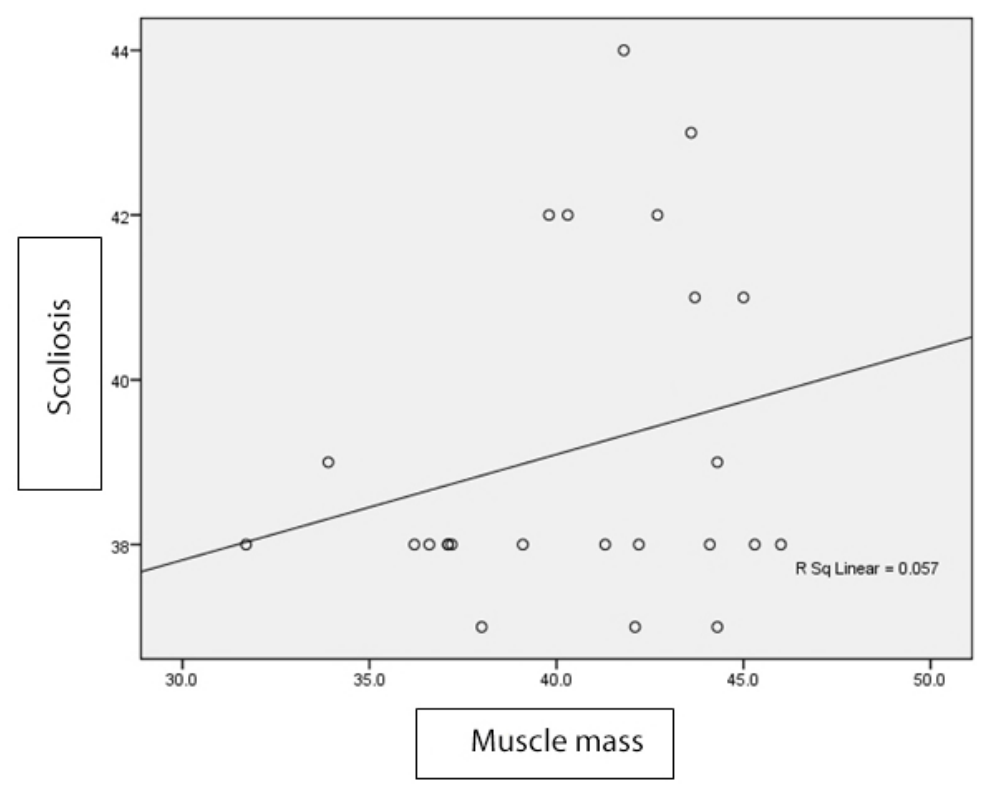

Figure1: The trend line graph between scoliosis and muscle mass

\section{Discussion and conclusions:}

This study was going to find out the correlation between anthropometric characteristics and skeletal malformations of scoliosis among male students of P.E. The results showed that there is no significant relationship between scoliosis and other factors. Dekle and his colleague's studies in 1996 showed the postural malformations which have relation with adolescent body(9), are inconsistent with the findings of this study. Rahmani \& his colleague's found out the scoliosis spine abnormalities associated with flexibility and BMI in girls with Down syndrome. The obtained results according to the interaction between body status, physical readiness and special needs that people with Down syndrome in performing their daily physical activities recommend the educators to give special attention to administration and implementation of reform programs, programs to improve the physical fitness \& weight control \& loss programs for these people. Deviation in body condition occurs when the asymmetrical and abnormal part of body cause the increase in muscle strain and impaired balance (8). Mirbagheri and his colleague's findings show that there is a significant difference between physical characteristics and body type with the prevalence of malformations of the spine based on the severity of the incidence and boys and girls pain tolerance that are consistent with Kargarfard and his colleague's study (10) and are inconsistent with this current study. 
Also, Guo and his colleagues in their research showed that the body mass index over $24 \mathrm{~kg}$ square mater or the ratio of waist to hip more than $85 \%$ may increase the size of the curvature of the spine (11).since the quite similar survey to the present study was not observed, therefore there may not be the possibility to compare the results of present study with other studies. If more researchers in relation to different statistical societies are done, the ambiguity in this position can be eliminated.

\section{References}

1. Letafatkar,O.,Daneshmandi,H.,Javadinejad,M.,Abdolrajaei0,Z.(2013).Advanced corrective movements of the body (second edition), Tehran: Avaye hozour.

2. Kashef,MM. (2006).corrective exercises \& physical therapy .Urumiyeh:University of urumiyeh.

3. Daneshmandi,M.,pourhosseini,H.,Sardar,MA. (2004). Comparative study of malformations of the body and girl student's spine. Harekat Publication, Issue 23 .PP:143-156.

4. Daneshmandi,H.,Alizadeh,MH., GHarakhanlou,R. (2011) .Corrective exercises (Tenth edition). Tehran .samt.

5. Farahani,A. (2011) the relationship between postural malformations of the spine with habits and consumables. Journal of Applied sport physiology No,13.PP:153-165.

6. Mahdavinejad,R. (2001). The prevalence of malformations of the spine among students of Isfahan University and the effects of the most common corrective exercise on them. Researchers project university of Isfahan.

7. Ahmadi,E. (2002). Checking the rates of spine malformations in male students at martyr Chamran university, MA thesis, Tehran, Tehran university of Tarbiat Moalem.

8. Rahmani,P.,shahroqi,H.,Daneshmandi,H. (2012). studying spine malformations associated with flexibility \& BMI in girls with Down syndrome. Research in Rehabilitation sciences No, 8.

9. Dekel ,Y; Gershon Tenenbaum; katalin kudar.( 1996). An Exploatory syudy on the relationship between postural Deformities and body- image and self - steam in adolescents: the Mediating role of physical Activity ; J. sport psychol; No.2. 27:183196.

10. Kargarfard,M.,Mahdavinejad,R., Ghasemi GA., Rouzbahani ,R., Ghias M., et al(2010) .measuring the curvature of the spine among students of Isfahan university . Journal of Medical sciences, No.27,Volume102,PP.762-776.

11. Guo.M and Zhang G.Q(2008). effect of BMI and WHR on lumber lordosis and sacrum slant angle in middle and elderly women = Zhongguo gu shang=china journal of orthopaedics and traumatology,21(1),30. 